\title{
Beneficios de un programa grupal de tratamiento no farmacológico en el afrontamiento del cáncer en mujeres españolas. Una síntesis cualitativa
}

\section{Benefits of a non-pharmacological treatment group program in coping with cancer in Spanish women. A qualitative synthesis}

\author{
Francisco-Xabier Aguiar Fernández \\ (D) https://orcid.org/0000-0001-5672-257X \\ E-mail: francisco.aguiarळuvigo.es

\section{Margarita Pino-Juste ${ }^{b}$} \\ (D) https://orcid.org/0000-0002-2551-5903 \\ E-mail: mpinoळuvigo.es

\section{José Javier Navarro Pérezc \\ (iD) https://orcid.org/0000-0001-6363-7154 \\ E-mail: j.javier.navarroळuv.es}

anniversidade de Vigo. Faculdade de Educación e Traballo Social. Departamento de Análise e Intervención Psicosocioeducativa. Ourense, Espanha.

bUniversidade de Vigo. Faculdade de Ciencias da Educación e do Deporte. Departamento de Didáctica, Organización Escolar e Métodos de Investigación. Pontevedra, Espanha.

'Universidad de Valencia. Faculdad de Ciencias Sociales. Departamento de Trabajo Social y Servicios Sociales. Valencia, Espanha.

\section{Correspondencia}

Francisco-Xabier Aguiar Fernández

Universidade de Vigo, Campus Universitário de Ourense.

As Lagoas, s/n, pabellon 2, 28 Vigo. Ourense, Espanha. CEP 36310.

\section{Resumen}

En el presente trabajo se analizan los beneficios de un programa grupal de tratamiento no farmacológico realizado por la Asociación Española contra el Cáncer en Galicia (España). Se analizaron 14 ediciones del programa en las que participaron 361 mujeres con cáncer de edades comprendidas entre los 33 y los 82 años. Se adoptó un diseño descriptivo de carácter etnográfico triangulando las técnicas de análisis y las fuentes de recogida de datos por medio de un cuestionario de satisfacción, 32 entrevistas en profundidad y un grupo de discusión con profesionales. Los resultados apoyan la idea de que el programa grupal analizado ha conseguido un elevado grado de satisfacción entre las mujeres participantes en todas las ediciones, ha favorecido la aceptación y el afrontamiento positivo de la enfermedad, aumentado los conocimientos y las habilidades para resolver los problemas relacionados con el proceso, así como promovido las relaciones sociales, la participación en la comunidad y el apoyo social. Desde el punto de vista práctico y profesional, nuestro estudio evidencia la importancia de diseñar e implementar programas grupales desde el ámbito psicosocial y con carácter interdisciplinar que permitan mejorar las estrategias de afrontamiento y el apoyo en poblaciones de mujeres diagnosticadas de cáncer. Palabras clave: Cáncer; Mujer; Programa Grupal; Tratamiento no Farmacológico; Beneficios. 
The study aims to determine the benefits of a nonpharmacological treatment and communitarian program carried out by the Spanish Association against Cancer in Galicia (Spain). The total of 14 editions of the program were analyzed in which 361 women with cancer between the ages of 32 and 84 participated. A descriptive ethnographic design was adopted, triangulating the analysis techniques and data collection sources using a satisfaction questionnaire, 32 in-depth interviews and a discussion group with professionals. The results support the idea that the group program analyzed has achieved a high degree of satisfaction among women participating in all editions, has favored the acceptance and positive coping with disease, increased knowledge and skills to solve related problems with the process, as well as promoted social relations, participation in the community and social support. From the practical and professional point of view, our research shows the importance of designing and implementing group programs from the psychosocial and interdisciplinary field that allow improving coping strategies and support in populations of women diagnosed with cancer.

Keywords: Cancer; Woman; Group Program; NonPharmacological Treatment; Benefits.
El cáncer es una de las enfermedades con mayor morbilidad y mortandad en las mujeres de todo el mundo, siendo el de mama el de mayor incidencia en España. Hoy en día tiende a cronificarse, por lo que se hacen necesarios abordajes multidisciplinares que, además de los aspectos biológicos, contemplen los psicológicos y sociales, tanto en el momento del diagnóstico y el tratamiento, como en la reincorporación a la vida cotidiana. El diagnóstico oncológico es acompañado con frecuencia de dificultades para la aceptación de la enfermedad, bajo estado de ánimo, depresión, miedos, angustia ante lo desconocido y sentimientos de pérdida (Cruzado, 2013; Fischer; Wedel, 2012; Yélamos et al., 2007). Las experiencias de los síntomas de los pacientes no ocurren aisladamente y deben ser abordadas de forma holística en su contexto de vida (Bennion; Molassiotis, 2013).

De hecho, algunos autores señalan que, si el distrés o malestar emocional durante la trayectoria de la enfermedad no es detectado y tratado en la etapa más temprana de la enfermedad, tiene un potencial efecto negativo sobre la morbilidad y la mortalidad, la cronificación, la calidad de vida y la adherencia y/o duración del tratamiento médico (Cruzado, 2013; Holland; Watson; Dunn, 2011). Entre los factores de riesgo psicosocial más habituales se encuentran las habilidades de afrontamiento pasivas, escaso apoyo social, temprana edad, la evitación de pensamientos relacionados con la enfermedad y una historia previa de problemas psicológicos (Cardenal et al., 2012; Fischer; Wedel, 2012; Rodríguez et al., 2007; Schnoll; Knowles; Harlow, 2002; Schroevers; Kraaij; Garnefski, 2011). Por el contrario, las emociones positivas y los estilos de afrontamiento más activos se relacionan con una mejor adaptación a la situación de enfermedad (Algoe; Stanton, 2012; Schnoll; Knowles; Harlow, 2002).

Todavía son escasos los estudios empíricos sobre la efectividad de las intervenciones psicosociales en personas con diagnóstico médico de cáncer. Muchos estudios indican que las personas diagnosticadas de cáncer participantes en intervenciones grupales obtienen beneficios 
especialmente relacionados con el estado afectivo y emocional (Beatty et al., 2018; Campbell; Phaneuf; Deane, 2004; Giese-Davis et al., 2006; Grégoire et al., 2017; Hoey et al., 2008; Iancu et al., 2017; Stevinson; Lydon; Amir, 2011); la reducción de la depresión y de la ansiedad (Cameron et al., 2007; Cohen; Fried, 2007; Merckaert et al., 2017); la mejora de la imagen corporal y autoestima (Esplen et al., 2018; Lewis-Smith et al., 2018); la adquisición de aprendizajes y estrategias de autocuidado y mejora del afrontamiento (Adams et al., 2019; Novakov et al., 2019; Ramos et al., 2018; Weis et al., 2020) o la mejora del bienestar general y la calidad de vida (Beatty et al., 2019; Grégoire et al., 2017; Lieberman; Goldstein, 2006; Santos; Souza, 2019). Estas personas señalan mejoras en aspectos como el funcionamiento emocional, las estrategias de afrontamiento, el conocimiento sobre el cáncer y sus tratamientos, las relaciones personales o la calidad de vida. Por otra parte, los grupos son espacios que permiten el intercambio de información, siendo este uno de los componentes más importantes para un adecuado soporte y cuidado (Fletcher et al. 2017; Turner et al. 2005). Una adecuada información tiene beneficios relacionados con una mayor involucración en las decisiones, una mayor adherencia a los tratamientos y menor ansiedad (D'haese et al., 200o; Husson; Mols; van de PollFranse, 2011).

El objetivo general de este estudio es evaluar los beneficios de un programa grupal de tratamiento no farmacológico con mujeres operadas de cáncer de mama que incluye una intervención profesional interdisciplinar. El programa fue realizado por la Asociación Española contra el Cáncer (AECC) en la comunidad autónoma de Galicia (España) entre los años 2004 y 2016, y está coordinado por el Departamento de Trabajo social de esta entidad. En concreto, en el presente artículo se analiza la satisfacción de las participantes con el diseño y contenidos del programa -la convivencia y la relación con el grupo y los beneficios percibidos en relación a la aceptación, el estado anímico y el afrontamiento de la enfermedad-; la adquisición de conocimientos y aprendizajes; las relaciones sociales; el apoyo social y la participación en la comunidad.

\section{Breve descripción del programa}

El programa consiste en una convivencia grupal de cuatro días. Su objetivo es que mujeres diagnosticadas de cáncer puedan interaccionar y compartir experiencias, resolver sus dudas, sobre todo de índole sociosanitaria, y descubrir nuevas posibilidades de cuidado personal y de relación con los demás. Tiene lugar en un centro ubicado en un entorno natural que invita al descanso y a recuperar fuerzas en contacto con la naturaleza y que reúne las instalaciones y recursos necesarios para el alojamiento, manutención y el desarrollo de las actividades.

Se trata de un programa en el que participan una media de 25 mujeres que incluye entre 11 y 12 sesiones grupales de una hora y media. Los contenidos abordan temáticas de afrontamiento de la enfermedad, tratamiento, efectos secundarios y rehabilitación, autocuidado de la salud, nutrición, autoestima e imagen corporal, sexualidad, comunicación y habilidades sociales y búsqueda de bienestar mediante la relajación, el yoga, la expresión corporal o la actividad física. El programa es coordinado por tres profesionales del trabajo social, pero para su desarrollo se cuenta con un equipo interdisciplinar de profesionales especializados en cada una de las temáticas (oncología médica, psicooncología, trabajo social, educación social, enfermería, nutricionista y terapeutas ocupacionales). La programación se intercala con espacios de tiempo libre donde las participantes pueden conversar, pasear por los jardines, descansar o promover otras actividades con carácter espontáneo. Las participantes son seleccionadas por profesionales de la entidad organizadora (psicólogos y trabajadores sociales), mediante entrevista previa, teniendo en cuenta su situación clínica y psicosocial, disponibilidad e interés en el programa. Pueden acudir por iniciativa propia o derivadas desde otros servicios de la misma entidad o de los servicios de salud y los servicios sociales, con los que existe una estrecha colaboración. Durante los seis meses siguientes, se organizan de dos a tres reuniones de seguimiento con carácter informal y las participantes mantienen al menos una entrevista de evaluación con una trabajadora social y una psicóloga de la entidad. 
Tabla I - Estructura básica y contenidos del programa en el período analizado de 2004 a 2016

\begin{tabular}{|c|c|c|}
\hline Temática & $\begin{array}{c}\mathrm{N}^{\circ} \\
\text { sesiones }\end{array}$ & Contenidos \\
\hline $\begin{array}{l}\text { Bienvenida/ } \\
\text { presentación }\end{array}$ & 1 & $\begin{array}{l}\text { Presentación. } \\
\text { Expectativas. } \\
\text { Establecimiento de } \\
\text { normas de grupo y de } \\
\text { convivencia. }\end{array}$ \\
\hline $\begin{array}{l}\text { Estrategias de } \\
\text { afrontamiento }\end{array}$ & 2 & $\begin{array}{l}\text { Repercusiones y } \\
\text { afrontamiento de la } \\
\text { enfermedad. Expresión } \\
\text { emocional e intercambio } \\
\text { de experiencias. }\end{array}$ \\
\hline $\begin{array}{l}\text { Autocuidado de la } \\
\text { salud }\end{array}$ & 1 & $\begin{array}{l}\text { Aspectos preventivos y } \\
\text { tratamiento de secuelas. } \\
\text { Cuidado y actividad física. }\end{array}$ \\
\hline $\begin{array}{l}\text { Alimentación y } \\
\text { nutrición }\end{array}$ & 1 & $\begin{array}{l}\text { Recomendaciones } \\
\text { para una adecuada } \\
\text { alimentación/nutrición. }\end{array}$ \\
\hline $\begin{array}{l}\text { Autoestima, imagen } \\
\text { corporal }\end{array}$ & 1 & $\begin{array}{l}\text { Impacto del diagnóstico } \\
\text { y de los tratamientos } \\
\text { en la autoestima. } \\
\text { Autocuidados estéticos. }\end{array}$ \\
\hline $\begin{array}{l}\text { Afectividad- } \\
\text { sexualidad }\end{array}$ & 1 & $\begin{array}{l}\text { Repercusiones en la } \\
\text { afectividad-sexualidad. } \\
\text { Estrategias y recursos. }\end{array}$ \\
\hline $\begin{array}{l}\text { Comunicación, } \\
\text { habilidades sociales } \\
\text { y empoderamiento } \\
\text { social }\end{array}$ & 1 & $\begin{array}{l}\text { Habilidades sociales, } \\
\text { relaciones con el entorno } \\
\text { y autonomía personal. } \\
\text { Conocimiento y ejercicio } \\
\text { de derechos. }\end{array}$ \\
\hline $\begin{array}{l}\text { Relajación, } \\
\text { expresión corporal } \\
\text { y actividad física }\end{array}$ & 3 & $\begin{array}{l}\text { Expresión corporal, } \\
\text { ejercicios de respiración, } \\
\text { yoga, juegos, danza y } \\
\text { risoterapia. }\end{array}$ \\
\hline $\begin{array}{l}\text { Despedida/ } \\
\text { evaluación }\end{array}$ & 1 & $\begin{array}{l}\text { Valoración grupal } \\
\text { e individual de la } \\
\text { experiencia. }\end{array}$ \\
\hline
\end{tabular}

\section{Metodología}

El estudio de caso se ha planteado desde un enfoque crítico con un diseño longitudinal descriptivo utilizando instrumentos de corte cualitativo
(Chittem 2014; Stake, 1998; Yacuzzi, 2005). Este planteamiento nos permite abordar la triangulación de técnicas y de fuentes de recogida de datos con el fin de contrastar la información recogida y evitar las posibles distorsiones en la interpretación de los hechos al considerar que cada persona tiene su peculiar forma de entender e interpretar la realidad (Taylor; Bogdan; Devault, 2015).

\section{Participantes}

En total se han analizado 14 ediciones del programa entre los años 2004 y 2016 en las que han participado 361 mujeres de edades comprendidas entre los 33 y los 82 años, con una edad media de 53,5 años. La mayoría casadas $(62,7 \%)$, separadas o divorciadas $(15,8 \%)$, solteras $(12,5 \%)$ y viudas ( $9 \%$ ). Un $57,3 \%$ tenía estudios primarios; el 24,5\% bachiller y el $18,3 \%$ estudios universitarios. En cuanto al perfil clínico, un 80,5\% fueron mujeres diagnosticadas de cáncer de mama y el 19,5\% restante de otro tipo de tumores (colorrectal, pulmón, estómago, útero-vagina, leucemia). En el momento inicial del programa, el 41,1\% seguían a tratamiento activo de quimioterapia o radioterapia y el 58,9\% los habían finalizado $(84,5 \%$ en los tres años previos).

\section{Instrumentos}

Los instrumentos utilizados fueron los siguientes:

Un cuestionario de satisfacción con el programa realizado a las 361 mujeres participantes que fue autoadministrado al término de cada edición. Este cuestionario fue elaborado por la propia institución promotora del programa siguiendo criterios que permitiesen valorar la satisfacción personal de las participantes y tener en cuenta sus propuestas de mejora. Consta de una parte de datos sociodemográficos y clínicos y de 21 preguntas con formato de respuesta en escala tipo Likert, donde 1 corresponde a "nada satisfecha/nada de acuerdo" y 5 a "muy satisfecha/totalmente de acuerdo". Contiene preguntas relacionadas con el grado de satisfacción con el diseño y la organización del programa (estructura, metodología, duración, atención recibida por los organizadores 
y profesionales, espacio y medios empleados...); los contenidos (interés e utilidad de las distintas sesiones y temáticas tratadas) y la interacción con el grupo (participación, valoración de las relaciones e integración con otras participantes en el grupo...).

Entrevistas en profundidad de carácter semiestructurado a 32 participantes en el programa realizadas entre marzo y junio de 2017, aleatoriamente entre las supervivientes de las 14 ediciones analizadas y asegurando un mínimo de 2-3 por cada edición. Las entrevistas fueron elaboradas y realizadas por el equipo de investigación del estudio. De acuerdo a los objetivos contemplados, se abordaron cuestiones relativas a la satisfacción con el programa, la relación e interacción con el grupo y los beneficios percibidos en la aceptación y afrontamiento de la enfermedad o la consecución y mantenimiento de relaciones posteriores. Se interrumpió la selección al detectar saturación en el análisis de datos.

Un grupo de discusión realizado en abril de 2017 en el que participaron 5 profesionales expertos que al menos hubiesen intervenido en 5 ediciones del programa (dos trabajadoras sociales, una psicooncóloga, una médica oncóloga y un terapeuta ocupacional). Se abordaron cuestiones relativas a la interacción grupal, los beneficios del programa en las participantes y la consecución y mantenimiento de relaciones posteriores.

\section{Procedimiento y análisis de los datos}

En relación a los aspectos éticos de la investigación se siguió el código deontológico reconocido por la Declaración de Helsinki (revisión de Hong-Kong, septiembre de 1989) y de acuerdo con las recomendaciones de Buena Práctica Clínica de la CEE (documento 111/3976/88 de julio de 1990) y la normativa legal vigente española que regula la investigación.

El proceso de recogida de datos se llevó a cabo a través de diferentes fuentes de información y se buscó la saturación de la misma para hacer válidos los resultados. Se ha contado con la colaboración de los profesionales del Departamento de Trabajo social de la Asociación Española contra el Cáncer en Galicia para la disponibilidad de los materiales (cuestionarios, programaciones y memorias). Así mismo, con su colaboración se ha podido acceder a las personas participantes en las entrevistas en profundidad y grupo de discusión que habían sido seleccionadas según los criterios establecidos, siendo posteriormente contactadas y entrevistadas por los autores del estudio. La participación fue voluntaria y se ha procedido a la firma de un consentimiento informado. Se ha mantenido la confidencialidad y anonimato en el tratamiento de los datos. Se han grabado y transcrito literalmente las entrevistas y el grupo de discusión, con una codificación identificativa de los participantes. La información recogida fue analizándose de forma paralela al propio proceso de trabajo de campo, como es habitual en los estudios cualitativos. El propósito era clasificar las diferentes partes de acuerdo con unas categorías previamente establecidas por lo que existe la posibilidad de identificar de manera sistemática y objetiva dichas categorías dentro de un mensaje. En consecuencia, el proceso se enmarca dentro de las técnicas de análisis de contenido cuyas fases han sido: lectura y comprensión de los textos, definición de las categorías, análisis de las mismas -selección e interpretación-y síntesis. De este modo emergieron las categorías principales de análisis que a continuación se detallan: satisfacción con el diseño y contenido del programa, la convivencia y la relación con el grupo, y los beneficios del programa en relación a la aceptación, el estado anímico y el afrontamiento positivo de la enfermedad; la adquisición de conocimientos y aprendizajes; las relaciones sociales, el apoyo social y la participación en la comunidad.

\section{Resultados}

Satisfacción con el programa, la convivencia y la relación con el grupo.

La valoración general del programa a su término se ha mantenido como satisfactoria o muy satisfactoria en porcentajes superiores al $94 \%$ en todas las ediciones, situándose la media en el $96,3 \%$. Un 97,3\% valoraron la relación con el grupo de compañeras como muy satisfactoria; un 98,3\% declararon haberse sentido a gusto en el grupo y la totalidad han manifestado que les gustaría seguir 
manteniendo contacto con alguna o varias personas del grupo. El programa cuenta con un proceso de evaluación en el que participan las mujeres de cada edición y en el que también se les pide que realicen propuestas de mejora. A pesar de los cambios que se han ido introduciendo en el diseño y los contenidos como consecuencia de este proceso, se ha podido identificar una estructura con bloques temáticos que se han mantenido a lo largo de la evolución del programa en el período analizado. En todas las ediciones se intercalaron actividades de tipo formativo/educativo y de tipo psicosocioterapéutico, con otras actividades más lúdicas, tiempos de descanso para las comidas que se comparten en grupo y tiempo libre para que las participantes aprovechen para pasear, descansar e interaccionar entre ellas. Los profesionales del programa que participaron en el grupo de discusión señalaron que el equilibrio en la programación entre las sesiones de carácter formativo y psicosocioterapéutico, y los contenidos de carácter lúdico y de tiempo libre, ha ido evolucionando a lo largo de los años. En las primeras tres ediciones los talleres suponían la mayor parte del tiempo programado en horario intenso de mañana y tarde. Sin embargo, en las valoraciones, las participantes han ido sugiriendo el deseo de disponer de más tiempo libre para conocerse y poder hablar de una manera más espontánea entre ellas. Como consecuencia, se incrementó el tiempo sin contenidos programados y las actividades de tipo lúdico. Una de las profesionales del grupo de discusión destacó: creo que el tiempo libre que tienen entre ellas acaba convirtiéndose en una actividad muy importante para que ellas se conozcan, compartan experiencias y se apoyen (GD. psicooncóloga). Otro factor destacado por los profesionales en el diseño del programa es el hecho de que se desarrolle en un espacio de convivencia en régimen de pensión completa y en un entorno natural distinto del habitual de las participantes. Se promueve así una convivencia que aleja a las mujeres de sus preocupaciones y rutinas cotidianas, propiciando un espacio para el autocuidado de ellas mismas, la relación con el grupo y el descanso. Otros aspectos importantes en la evaluación del diseño por el equipo de profesionales es el hecho de que los grupos se mantengan en un tamaño no superior a
25-26 personas; que sean heterogéneos en edades y situaciones clínicas y el especial cuidado que se ha de tener en la atención y acompañamiento profesional previo, durante y posterior a la estancia.

Entre las dificultades surgidas, en las entrevistas en profundidad, casi un cuarto de las mujeres afirmaron haberse sentido ansiosas o con miedo a no lograr relacionarse o a sentirse aisladas en el grupo en los días anteriores a la participación en el programa, incluso después de haber sido informadas y entrevistadas por los profesionales y de haber decidido asistir.

Dije que sí, pero tenía miedo a lo que me iba a encontrar, mi mundo era mi casa, nunca había participado en algo así, no sabía cómo me iba a sentir. (E. 6)

Para algunas mujeres no es fácil salir de casa durante cuatro días, yo tenía hijos pequeños. Ir allí, enfrentarte tú sola, no conocer a nadie, no saber si te vas a integrar en el grupo, te retrotrae al principio. (E. 13)

Al principio no quería ir porque nunca había salido así en grupo, pero después cuando fui no quería que se terminase. (E. 25)

Sin embargo, en todas las entrevistas se ha podido constatar una elevada valoración de la convivencia grupal independientemente del tiempo transcurrido desde la participación en el programa, así como la presencia de relaciones positivas con el grupo o con algunas de las mujeres en particular. El 80\% de las participantes entrevistadas valoró tener la oportunidad de conocer e intercambiar experiencias con personas con las que se comparte un mismo problema y preocupaciones. Han sido predominantes los sentimientos deautoidentificación, comprensión mutua y unión con el grupo. El grupo es concebido como un espacio donde poder hablar y expresarse con libertad, incluso en casos de personas que no habían conseguido verbalizar o expresarse abiertamente con anterioridad.

Me encontrémuy a gusto con la gente, el sitio... pude expresarme, decirmis pesares, escuché a las demás... El contacto con la gente fue una maravilla, compartir con la gente que está en tu situación. (E. 12) 
Cuando llegas allíves que hay gente muy distinta, pero es como si en ese momento todas nos sintiéramos identificadas. Claro que yo al principio tenía miedo de lo que me iba a encontrar, y claro que al principio te cuesta más abrirte, pero lo bueno es que nadie está obligado a hablar de símismo, y que lo más importante es el respeto a cada una de nosotras, eso nos lo dejan claro desde el inicio, y al final todo fluye muy bien. No tienes por qué compartir todas las opiniones, ni tampoco te identificas por igual con todo el mundo. (E. 19)

En más de un tercio de las entrevistas se explicitó que en el entorno familiar o red social habitual no se habían sentido igual de comprendidas. Entre los motivos, se han señalado el no querer o no poder hablar abiertamente de sus propios sentimientos para no preocupar a la familia y a los entornos habituales, el haberse sentido sobreprotegidas por familiares y amistades o por el contrario, haberse sentido decepcionadas por no haberles permitido expresarse o no haberles mostrado interés y comprensión ante sus vivencias. Incluso en los casos de las mujeres que se han sentido acompañadas y que consideran que cuentan con una buena red social de apoyo, se ha podido observar cierto grado de "soledad emocional" previa relacionada con el hecho de no conocer a gente en situaciones similares y no poder expresarse abiertamente con otras personas que, por el contrario, sí han visto satisfecho en el programa.

A lo mejor en tu casa no hablas de esto, no quieres que tu dolor vaya hacia ellos, con la gente igual que tú parece que te abres, que te encuentras mejor solo con hablar. (E. 7)

La familia a veces trata de sobreprotegerte y uno no quiere, más bien te gustaría que te tratasen normal, no como si fueses una cosa frágil que se va a romper. (E. 18)

Me sentí sola muchas veces, porque estaba sin mi familia aquí...hubo un momento en que me metí en cama y no quería levantarme... el grupo ayuda mucho, porque uno se siente dentro de una familia, porque me trataron como una más. (E. 15)
En el grupo de discusión de profesionales se valoró muy positivamente el progreso en la interacción y la cohesión observadas en todas las ediciones a pesar de que la mayor parte de las mujeres no se conocían entre sí previamente, observando cambios muy perceptibles entre el primer y el último día de la convivencia, e incluso tras las primeras horas. Estos cambios se han producido en grupos que como ya se ha comentado presentan variables sociodemográficas y situaciones clínicas heterogéneas. En el grupo de discusión también se identificaron casos en los que la integración presentó más dificultades debido al estado anímico y de salud, pero generalmente el propio grupo funciona como soporte que ayuda a superar estas situaciones. Aunque no se han detectado situaciones de aislamiento o no integración en el grupo, los profesionales señalan que algunas participantes, principalmente las personas en peores situaciones anímicas, con personalidades más introvertidas o que non han participado en experiencias grupales previas, necesitan de una especial observación y acompañamiento individual complementario que les facilite la incorporación al programa. Por otra parte, las principales dificultades señaladas por las mujeres durante la estancia también tienen relación con los miedos a lo desconocido y a la integración inicial en el grupo. Algunas mujeres también han señalado la dificultad de estar fuera de casa durante unos días, si bien generalmente esto es señalado como una oportunidad de desconectar de las obligaciones cotidianas. En este sentido, una buena información y acompañamiento profesional de carácter individual en la selección, así como durante el programa, especialmente en estos casos, es uno de los factores principales que aminora los posibles inconvenientes derivados de la intervención grupal.

Se ve un cambio que experimentan en horas desde el inicio del programa, incluso en personas que en un principio les cuesta más comunicarse o participar. (GD. Educadora social)

La gente necesita espacios de relación, compartir experiencias, salir de sus rutinas, del aislamiento, creo que el espacio natural que se comparte durante unos días influye en que se acelere la cohesión 
del grupo, me ha sorprendido mucho como en tan poco tiempo realmente viven la experiencia con intensidad y compartiendo. (GD. Psicooncóloga)

Cuidamos mucho el momento de la selección de las participantes, asegurándonos que comprendan bien los objetivos del programa, resolviendo sus dudas o miedos iniciales, generalmente miedo a lo que se van a encontrar allí, a si no se van a integrar en el grupo [...] en algunos casos es necesario motivarlas, pero también estamos atentos y sivemos que una persona no está preparada para participar es preferible respetar los tiempos. (GD. Trabajadora social)

Solamente hemos tenido dos casos de abandono del programa, en una ocasión una mujer se tuvo que ir al segundo día por un problema familiar y otra porque no se encontraba bien de salud, pero ninguno fue por falta de integración en el grupo, eso tratamos de trabajarlo en el proceso de selección de participantes $y$ en los primeros momentos de la estancia. Tratamos de cuidar cada detalle en la convivencia, desde la comida a los talleres, y los profesionales convivimos y somos parte del programa. (GD. Psicooncóloga)

\section{Beneficios en la aceptación, el estado anímico y el afrontamiento positivo de la enfermedad}

Para muchas participantes el programa propició la mejora de su estado de ánimo. Antes del programa, muchas refirieron haberse sentido solas, deprimidas o incomprendidas; no aceptar la situación; no ser capaces de hablar con otras personas de su enfermedad y aislarse socialmente.

Cuando me diagnosticaron el mundo se me vino abajo, me estaba separando, todo al mismo tiempo, o salía o me estancaba, el programa fue como un punto de inflexión. (E. 2)

Me sentía muy sola porque a míme diagnosticaron la enfermedad a los dos meses de llegar a España..., la verdad no sabía qué hacer. (E. 13)

La mayoría de las entrevistadas señalaron que la participación en el programa les había ayudado a aceptar mejor la enfermedad. Entre las percepciones más señaladas están que el programa contribuye a sentirse más seguras, darse cuenta de que no eran las únicas, poder hablar de sus sentimientos con mayor facilidad y querer luchar y salir adelante con mayor optimismo y esperanza. Así mismo, que la experiencia grupal les sirvió para mejorar su autoestima y aprender a pensar más en una misma.

Hablando con la gente, escuchándoles, te das cuenta que no eres la única. (E. 28)

Me di cuenta que tenía que luchar, que cada uno tiene que enfrentar su vida y su enfermedad. (E. 5)

Me hizo aceptar la enfermedad con más optimismo y vivirla de otra manera. (E. 14)

Hablamos mucho entre nosotras esos días, yo vi gente que veías que después de allí iba a ver todo diferente... los miedos... que desde ese momento se los iban a replantear. (E. 8).

Tuve muchos momentos de bajón, pero el programa me ayudó mucho, fue como un empujón para salir adelante con más fuerza y quererme más a mí misma. (E. 23)

En los grupos confluyen personas con distintas características sociodemográficas, situaciones anímicas y de salud, y estilos de afrontamiento, lo que parece favorecer los beneficios mutuos. A la hora de valorar el intercambio de experiencias, destaca el elemento positivo de la reciprocidad. En un tercio de las entrevistas se señaló que el programa les ha permitido ver que existen situaciones distintas a las suyas, en algunos casos peores (principalmente por edad más temprana en el diagnóstico, situación clínica, física o emocional). A este respecto, el grupo no solo les dio la posibilidad de sentirse escuchadas, sino de poder ayudar a otras personas a través de su propio testimonio y ayuda.

En el grupo ves que lo que te pasa a ti también le pasa a otras personas, intercambias experiencias... Al ser personas que venían de distintos ámbitos, edades, situaciones médicas... ves que aportaban y que tú 
también aportabas, creo que eso nos da confianza en nosotras mismas y nos hace sentirnos mejor. (E. 3)

\section{Beneficios relacionados con la adquisición de conocimientos útiles para el manejo de la enfermedad y su tratamiento}

Los contenidos de las actividades fueron valorados satisfactoriamente en los cuestionarios por un $82,3 \%$ del total de participantes. En todas las ediciones, las sesiones de tipo formativo de carácter biomédico y psicosocial recibieron valoraciones satisfactorias o muy satisfactorias superiores al $80 \%$. Para un $83,9 \%$ se cumplieron sus expectativas iniciales con los contenidos. La información recibida en el grupo, tanto por parte de los profesionales como de las otras personas participantes es muy importante. Resulta especialmente valorada la información práctica sobre los tratamientos, las secuelas y la rehabilitación, la alimentación, la psicoafectividad y sexualidad, así como el haber podido resolver dudas con expertos. Por su parte, en las entrevistas, todas las mujeres comentaron que el programa les había ayudado a mejorar sus conocimientos y que estos fueron útiles para el afrontamiento de la enfermedad, independientemente de la edición y el tiempo transcurrido desde su participación. En más de la mitad de los casos afirmaron que existen gran cantidad de temas relacionados con la enfermedad del que no reciben suficiente información por parte de los equipos médicos y de salud (sobre todo a un nivel práctico), que han podido descubrir, contrastar o debatir en el programa. Los contenidos formativos y educativos tratados en el grupo han fortalecido aspectos como la sensación de seguridad y el control de la sintomatología de la enfermedad y los tratamientos, la adopción de nuevas estrategias preventivas o el incremento del bienestar, por ejemplo, a través de la prevención de efectos secundarios de los tratamientos, la mejora de la alimentación o la práctica de ejercicio y actividad física.

Yo siempre estaba en casa...ahora me propuse salir a caminar todos los días y hacer ejercicio... desde entonces sé lo que es hacer algo por mí y para mí. (E. 6)
Antes no me daba cremas, sin embargo ahora hago un cuidado de la piel necesario. (E. 17)

Resuelves muchas dudas sobre cuestiones de salud, de nutrición, de sexualidad o de cómo cuidarnos que no te explican en la consulta. (E. 26)

\section{Beneficios en las relaciones sociales, la participación en la comunidad y el apoyo social.}

Entre los beneficios del programa también se han descrito la adquisición de mayores habilidades de comunicación y de relación con el entorno. Más de la mitad de las mujeres entrevistadas señalaron que el programa les ayudó a abrirse más a otras personas y tener más facilidades para relacionarse con gente desconocida. Incluso hay mujeres que refieren que solo fueron abiertas en los días y con las personas del programa porque en aquel momento se encontraban en un espacio de confianza y con gente desconocida hasta el momento, lo que paradójicamente podría haber facilitado la expresión de sentimientos y el intercambio de experiencias.

Alli he visto por ejemplo una chica muy tímida que al acabar dio un cambio grandísimo, al principio no hablaba casi y al final se abrió mucho, creo que lo necesitaba. (E. 1)

El programa hizo que me abriese más, que sea más extrovertida, que logre expresar mejor mis sentimientos. (E. 14)

En el momento que estás allí te liberas mucho, yo solo fui abierta allí, con el paso del tiempo fui volviendo a mi vida, pero aquella experiencia la tengo guardada en un rinconcito con mucho cariño. (E. 20)

Esta mejora en la comunicación también se manifiesta en el entorno familiar o a la hora de relacionarse o plantear dudas al equipo médico.

Hablar de ciertos temas o preocupaciones con mi marido o mis hijos no es fácil, pero creo que aprendí a hablarles de mis deseos con mayor facilidad, a pensar un poco más en mí, en mis necesidades. (E. 11) 
Me sirvió para tener más seguridad en mí misma, sobre lo que hacer con mi enfermedad... y cuando iba al médico ya empecé a preguntarle, a decirle... porque antes no me atrevía... me ayudó mucho. (E. 29)

En el grupo de profesionales también se destacaron los cambios en la manera de relacionarse observados en algunas mujeres que no estaban acostumbradas a participar en grupos y lo especialmente beneficioso que resulta en casos de tener dificultades en las habilidades sociales o para entablar relaciones sociales, bajo estado anímico o escasa red de apoyo social.

Obviamente no todas las mujeres llegan al programa en las mismas circunstancias. A algunas les cuesta más expresarse o relacionarse con el grupo porque son más introvertidas o están anímicamente peor, pero en esos casos es donde ves a menudo los cambios que más sorprenden y lo beneficioso que puede llegar a ser el programa. Hemos tenido casos de personas que por primera vez hablaban allí de sus miedos, de sus dudas [...] Hay personas que viven en zonas rurales o que tienen a sus familiares y amigos en otros países con una red social de apoyo muy limitada, que no habían conocido a personas en situaciones similares, que realmente no tenían nadie con quien compartir lo que les ofrece un espacio como este. (GD. trabajadora social 2)

El programa también ha evidenciado un incremento de la participación social en la comunidad, perceptible con la realización de nuevas actividades y encuentros realizados por iniciativa de las participantes con posterioridad. A través de las entrevistas se han identificado en todos los grupos, en mayor o menor medida, la organización de encuentros y actividades de manera autónoma (comidas o cenas, actividades de ocio, excursiones...), así como una mayor participación en otras actividades de la propia asociación organizadora u otras de las comunidades de referencia.

A partir de ahí para mí fue el despegue, empecé a relacionarme más. (E. 7)
Ahora participo en más actividades que antes, en la asociación y fuera de ella, y sigo en contacto con muchas personas del grupo. (E. 16)

Con posterioridad al programa se han mantenido redes de relación y apoyo, en sus distintos niveles e intensidades, favorecidas en las últimas ediciones por el uso de las redes sociales y las aplicaciones como WhatsApp que han ofrecido acompañamiento no solo emocional, sino también físico e instrumental. Estas redes se han mantenido activas con el paso del tiempo, especialmente durante los dos o tres años posteriores al programa. Ofrecen apoyo emocional a las personas del grupo que tienen problemas personales, que recaen o que empeoran su estado de salud física, pero también se han descrito acompañamientos a pruebas médicas, visitas en hospitalizaciones prolongadas o al domicilio de miembros del grupo en casos de recaídas. Algunas personas que se han conocido en el grupo pasan a formar parte de la red de amistades íntimas de las mujeres. En otros casos, no se mantiene el contacto o este se va perdiendo paulatinamente con el paso del tiempo, la reincorporación a la vida cotidiana o por considerar que es una etapa que prefieren dejar atrás. Incluso en estos casos, la valoración de la experiencia grupal se mantiene como positiva y se reconocen los beneficios personales en la aceptación y afrontamiento de la enfermedad.

Siempre conectas más con unas personas que con otras, yo mantengo mucha amistad con dos personas del grupo. (E. 4)

Seguimos teniendo un grupo de WhatsApp, seguimos en contacto desde siempre y nos vemos bastante, todos los años procuramos juntarnos las que podemos en algún lugar. (E. 19)

Las redes sociales contribuyen a mantenernos en contacto con la gente de más lejos. Yo vivo en una aldea y si no fuese por eso sería muy difícil. (E. 30)

Creo que logran apoyarse unas a otras [...] no solo con la enfermedad, sino muchas veces también a nivel personal. Muchas veces nos hablan de sus reuniones, de cómo siguen en contacto, se visitan 
y apoyan, y no solo cuando alguna lo necesita, las buenas noticias las acaban también compartiendo. (GD. Trabajadora social 1)

\section{Discusión}

Los resultados de nuestro estudio sugieren que una intervención grupal de carácter interdisciplinar para mujeres con cáncer, ofrecida en un entorno de convivencia comunitario, les beneficia en términos de afrontamiento positivo de la enfermedad y mejora del bienestar. Otras intervenciones grupales, aunque han utilizado metodologías diferentes, sugieren beneficios similares (Beatty et al., 2018; Cameron et al., 2007; Campbell et al., 2004; Cohen; Fried, 2007; Giese-Davis et al., 2006; Grégoire et al., 2017; Hoey et al., 2008; Iancu et al., 2017; Lieberman; Goldstein, 2006; Stevinson; Lydon; Amir, 2011).

Se ha podido constatar una elevada satisfacción con el programa, con la convivencia y la integración grupal, y el establecimiento de relaciones positivas entre las participantes. Se ha destacado la prevalencia de sentimientos de autoidentificación y comprensión mutua que favorecen el poder expresarse y hablar abiertamente, el intercambio de experiencias y la ayuda mutua. El apoyo percibido se aprecia como distinto al recibido en los entornos familiares y en la red social habitual (Ussher et al., 2006).

El programa ha evidenciado buenos resultados en la adquisición de estilos y estrategias de afrontamiento positivos ante la enfermedad, ya que posibilita contactar con distintos estilos de afrontamiento, estrategias de autocuidado y de relación social. Las participantes manifestaron mayoritariamente que el programa había favorecido su aceptación de la enfermedad, el espíritu de lucha, el optimismo y la esperanza, y, en general, el tener una actitud más positiva ante la misma. En sintonía con la literatura existente, la aceptación y los estilos de afrontamiento más positivos se asocian con una mejor adaptación a la situación de enfermedad (Schnoll; Knowles; Harlow, 2002).

El programa también ha permitido la adquisición de nuevos conocimientos sobre la enfermedad y los tratamientos y el intercambio de información en aspectos físicos, psicológicos y sociales, favoreciendo así la formación y el entrenamiento en estrategias que pueden ayudar a asumir de una manera más positiva el proceso, manejar los síntomas inesperados y asumir la sintomatología crónica (Bennion; Molassiotis, 2013; Ramos et al., 2018; Weis et al., 2020). Disponer de una adecuada información que incluya varios elementos (Fletcher et al., 2017) es uno de los aspectos más valorados por nuestras participantes, relacionándose con un mayor control del tratamiento, el autocuidado y menor ansiedad (D'haese et al., 200o; Husson; Mols; van de Poll-Franse, 2011; Turner et al., 2005).

Por último, el programa contribuyó a incrementar las relaciones sociales, la participación y la generación de nuevas redes sociales de apoyo con continuidad. Algunos grupos han logrado autogestionarse con posterioridad y constituirse en una red que ofrece apoyo emocional, informativo e incluso instrumental a sus miembros. Este aumento de las redes sociales y el apoyo social ha sido relacionado en diversos estudios con una mayor calidad de vida después del diagnóstico del cáncer y contribuye a evitar los problemas de aislamiento y soledad (Embuldeniya et al., 2013; Hasson-Ohayon et al., 2016; Howard et al., 2014; Ikeda et al., 2013; Kroenke et al., 2013; Steptoe et al., 2013; Tilvis et al., 2012).

La comparación con estudios previos se ve limitada por tratarse de propuestas de intervención grupal con diseños, contenidos y evaluaciones divergentes, aplicados además en situaciones y poblaciones con características sociodemográficas y clínicas dispares. Sin embargo, nuestra investigación permite extraer algunas interesantes conclusiones con respecto a la intervención con grupos de mujeres operadas de cáncer de mama. Se han podido identificar temáticas de interés y una estructura en la organización y contenidos del programa que se ha mantenido en el período analizado. Aspectos como la heterogeneidad de los grupos; la combinación de sesiones de tipo formativo (biomédico, psicosocial, autocuidados...) con otras de tipo lúdico y tiempo libre; la convivencia intensiva en un espacio natural entre las participantes y los profesionales o el cuidado de la atención profesional y el acompañamiento previo, durante y después del programa, parecen ser algunos de los factores que se relacionan con los buenos resultados de este programa. 
También queremos destacar que este estudio permitió por primera vez en una población española analizar un programa grupal teniendo en cuenta un significativo número de ediciones y participantes, lo cual pone de relieve que los resultados se han mantenido a partir de un diseño similar de la intervención grupal en el período analizado. Sin embargo, hemos de señalar entre las limitaciones de nuestro estudio, el hecho de que no se hayan aplicado instrumentos de medida pre-post test, y la necesidad de ampliar estos resultados con la utilización de grupos de control o con la evaluación de intervenciones específicas en relación a las características sociodemográficas y clínicas de las participantes, tratando de avanzar en los diseños y criterios que puedan resultar más idóneos en cada situación.

\section{Consideraciones finales}

En conclusión, nuestro estudio ha detectado una elevada satisfacción de las mujeres con cáncer de mama con una intervención grupal de carácter interdisciplinar, autopercibiendo beneficios en relación a la aceptación, el estado anímico y el afrontamiento de la enfermedad; la adquisición de conocimientos y aprendizajes de carácter biomédico, psicosocial y autocuidados útiles para la comprensión y el abordaje de la enfermedad y sus secuelas, además de propiciar las relaciones sociales, el apoyo social y la participación en la comunidad.

Teniendo en cuenta que este tipo de intervenciones siguen siendo minoritarias en el contexto español, señalamos la importancia de avanzar en el diseño, implementación e investigación de programas grupales desde el ámbito psicosocial que permitan mejorar las estrategias de afrontamiento y el apoyo en poblaciones de mujeres diagnosticadas de cáncer.

\section{Referencias}

ADAMS, L. et al. Effectiveness of a motivationalvolitional group intervention to increase physical activity among breast cancer survivors compared to standard medical rehabilitation-Study protocol of a prospective controlled bi-centred interventional trial. European Journal of Cancer Care, Hoboken, n. 28, n. 4, p. e13073, 2019.

ALGOE, S. B.; Stanton, A. L. Gratitude when it is needed most: Social functions of gratitude in women with metastatic breast cancer. Emotion, Worcester, v. 12, n. 1, p. 163-168, 2012.

BEATTY, L. et al. A systematic review of psychotherapeutic interventions for women with metastatic breast cancer: Context matters. Psycho-Oncology, Hoboken, n. 27, n. 1, p. 34-42, 2018.

BENNION, A. E.; Molassiotis, A. Qualitative research into the symptom experiences of adult cancer patients after treatments: a systematic review and meta-synthesis. Supportative Care Cancer, Berlim, v. 21, n. 1, p. 9-25, 2013.

CAMERON, L. D. et al. Changes in emotion regulation and psychological adjustment following use of a group psychosocial support program for women recently diagnosed with breast cancer. Psycho-Oncology, Hoboken, v. 16, n. 3, p. 171-18o, 2007.

CAMPBELL, H. S.; Phaneuf, M. R.; Deane, K. Cancer peer support programs-do they work? Patient Education and Counselling, Amsterdã, v. 55, n. 1, p. 3-15, 2004.

CARDENAL, V. et al. Personality, emotions and coping styles: predictive value for the evolution of cancer patients. The Spanish journal of psychology, Madri, v. 15, n. 2, p. 756-67, 2012.

CHITTEM, M. Understanding coping with cancer: How can qualitative research help? Journal of Cancer Research and Therapeutics, Nova Deli, v. 10, n. 1, p. 6-10, 2014.

COHEN, M.; Fried, G. Comparing relaxation training and cognitive-behavioral group therapy for women with breast cancer. Research on Social Work Practice, Thousand Oaks, v. 17, n. 3, p. 313-323, 2007.

CRUZADO, J. A. Manual de psicooncologia. Madri: Pirámide, 2013.

D'HAESE, S. et al. The effect of timing of the provision of information on anxiety and satisfaction of cancer patients receiving 
radiotherapy. Journal Cancer Education, Thousand Oaks, v. 15, n. 4, p. 223-227, 2000.

EMBULDENIYA, G. et al. The experience and impact of chronic disease peer support interventions: a qualitative synthesis. Patient Education and Counselling, Amsterdã, v. 92, n. 1, p. 3-12, 2013.

ESPLEN, M. J. et al. Restoring body image after cancer (ReBIC): results of a randomized controlled trial. Journal of Clinical Oncology, Alexandria, n. 36, n. 8, p. 749-756, 2018.

FISCHER, D.; Wedel, B. Anxiety and depression disorders in cancer patients: incidence, diagnosis and therapy. Memo-Magazine of European Medical Oncology, Viena, v. 5, n. 1, p. 52-54, 2012.

FLETCHER, C. et al. The information needs of adult cancer survivors across the cancer continuum: A scoping review. Patient $E d u c a t i o n$ and Counselling, Amsterdã, v. 10o, n. 3, p. 383-410, 2017.

GIESE-DAVIS, J. et al. Emotional expression and diurnal cortisol slope in women with metastatic breast cancer in supportive-expressive group therapy: a preliminary study. Biological psychology, Amsterdã, v. 73, n. 2, p. 19o-198, 2006.

GRÉGOIRE, C. et al. Group interventions to reduce emotional distress and fatigue in breast cancer patients: a 9-month follow-up pragmatic trial. British Journal of Cancer, Londres, n. 117, n. 10, p. 1442-1449, 2017.

HASSON-OHAYON, I. et al. The need for friendships and information: dimensions of social support and posttraumatic growth among women with breast cancer. Palliative supportive care, Cambridge, v. 14, n. 4, p. 387-392, 2016.

HOEY, L. M. et al. Systematic review of peersupport programs for people with cancer. Patient Education and Counselling, Amsterdã, v. 70, n. 3, p. 315-37, 2008.

HOLLAND, J.; Watson, M.; Dunn, J. The IPOS new International Standard of Quality Cancer Care: integrating the psychosocial domain into routine care. Psycho-Oncology, Hoboken, v. 2o, n. 7, p. 677-8o, 2011.
HOWARD, A. F. et al. Trajectories of social isolation in adult survivors of childhood cancer. Journal of Cancer Survivorship, Nova York, v. 8, n. 1, p. 80-93, 2014.

HUSSON, O.; Mols, F.; Van de Poll-Franse, L.V. The relation between information provision and health-related quality of life, anxiety and depression among cancer survivors: a systematic review. Annals of Oncology, Boston, v. 22, n. 4, p. 761-72, 2011.

IANCU, M. et al. The effect of psychological group intervention on emotional problems, event impact and quality of life in breast cancer patients under radiotherapy: a pilot study. Journal of EvidenceBased Psychotherapies, Cluj-Napoca, v. 17, n. 2, p. 133-146, 2017.

IKEDA, A. et al. Social support and cancer incidence and mortality: the JPHC study cohort II. Cancer Causes Control, Oxford, v. 24, n. 5 , p. 847-86o, 2013.

KROENKE, C. H. et al. Social networks, social support, and burden in relationships, and mortality after breast cancer diagnosis in the Life After Breast Cancer Epidemiology (LACE) study. Breast Cancer Research and Treatment, Boston, v. 137, n. 1, p. 261-271, 2013.

LEWIS-SMITH, H. et al. Efficacy of psychosocial and physical activity-based interventions to improve body imageamong women treated for breast cancer: a systematic review. PsychoOncology, Hoboken, v. 27, n. 12, p. 2687-2699, 2018.

LIEBERMAN, M. A.; Goldstein, B. A. Not all negative emotions are equal: the role of emotional expression in online support groups for women with breast cancer. Psycho-Oncology, Hoboken, v. 15, n. 2, p. 16o-168, 2006.

MERCKAERT, I. et al. Improving anxiety regulation in patients with breast cancer at the beginning of the survivorship period: a randomized clinical trial comparing the benefits of single-component and multiple-component group interventions. Psycho-Oncology, Hoboken, n. 26, n. 8, p. 1147-1154, 2017. 
NOVAKOV, I. et al. Empirical validation of the integrative psychological group intervention for women with breast cancer - preliminary results. Vojnosanitetski Pregled, Belgrado, v. 76, n. 10, p. 1022-1028, 2019.

RAMOS, C. et al. The effectiveness of a group intervention to facilitate posttraumatic growth among women with breast cancer. Psych-Oncology, Hoboken, n. 27, p. 258-264, 2018.

RODRÍGUEZ, B. et al. Adaptación individual y depresión en una muestra de pacientes oncológicos. Psicooncología, Madri, v. 1, n. 4, p. 7-19, 2007.

SANTOS, M. A.; SOUZA, C. Group interventions for women with breast cancer: Challenges and possibilities. Psicologia: Teoria e Pesquisa, Brasília, DF, v. 35, p. e5410, 2019.

SCHNOLL, R. A.; Knowles, J. C.; Harlow, L. Correlates of adjustment among cancer survivors. Journal of Psychosocial Oncology, Filadélfia, v. 8, n. 20, p. 37-59, 2002.

SCHROEVERS, M. J.; Kraaij, V.; Garnefski, N. Cancer patients' experience of positive and negative changes due to the illness: relationships with psychological well-being, coping, and goal reengagement. Psycho-Oncology, Hoboken, v. 20, n. 2, p. 165-172, 2011.

STAKE, R. E. Investigación con estudio de casos. Madri: Ed. Morata, 1998.

STEPTOE, A. et al. Social isolation, loneliness, and all-cause mortality in older men and women. PNAS, Washington, DC, v. 110, n. 15, p. 5797-5801, 2013. Disponível em: <https://bit.ly/2AgUWUj>. Acesso em: 11 jun. 2020.
STEVINSON, C.; Lydon, A.; Amir, Z. Cancer support group participation in the United Kingdom: a national survey. Supportative Care Cancer, Berlim, v. 19, n. 5, p. 675-683, 2011.

TAYLOR, S. J.; Bogdan, R.; DeVault, M. Introduction to qualitative research methods: a guidebook and resource. Hoboken: John Wiley \& Sons, 2015 .

TILVIS, R. S. et al. Social isolation, social activity and loneliness as survival indicators in old age; a nationwide survey with a 7-year follow-up. European Geriatric Medicine, Amsterdã, v. 3, n. 1, p. 18-22, 2012.

TURNER, J. et al. Clinical practice guidelines for the psychosocial care of adults with cancer. PsychoOncology, Hoboken, v. 14, n. 3, p. 159-173, 2005.

USSHER, J. et al. What do cancer support groups provide which other supportive relationships do not? The experience of peer support groups for people with cancer. Social Science Medicine, Nova York, v. 62, n. 1o, p. 2565-2576, 2006.

WEIS, J. B. et al. Psychoeducational group intervention for breast cancer survivors: a non-randomized multi-center pilot study. Supportive Care in Cancer, Berlim, v. 28, n. 7, p. 3033-3040, 2020.

YACUZZI, E. El estudio de caso como metodología de investigación: teoría, mecanismos causales, validación. Buenos Aires: Universidad del CEMA, 2005. (Serie Documentos de Trabajo, n. 296). Disponível em: <https://bit.ly/2AfGoWt $>$. Acesso em: 11 jun. 2020.

YÉLAMOS, C. et al. "Mucho x Vivir". Atención psicosocial para mujeres con cáncer de mama. Psicooncología, Madri, v. 4, n. 2, 3, p. 417-422, 2007.

\section{Contribución de los autores}

Los tres autores han participado conjuntamente en el diseño y planteamiento del estudio, análisis de datos y redacción final.

Recibido: 27/05/2019

Re-presentado: 21/03/2020

Aprobado: 25/05/2020 